\title{
A Robotic Torso Joint with Adjustable Linear Spring Mechanism for Natural Dynamic Motions in a Differential-Elastic Arrangement
}

\author{
Jens Reinecke ${ }^{1}$, Alexander Dietrich ${ }^{1}$, Anton Shu ${ }^{1}$, Bastian Deutschmann ${ }^{1}$, and Marco Hutter ${ }^{2}$
}

\begin{abstract}
To be operated in unknown or complex environments, modern robots have to fulfill various challenging criteria. Among them, one finds requirements such as a high level of robustness to withstand impacts and the capabilities to physically interact in a safe manner. One way to achieve that is to integrate variable-stiffness actuators into the systems, enabling compliant behavior through the elastic components and providing the additional adaptability of the impedance. Here, we introduce a novel adjustable linear stiffness joint mounted in a differential-elastic arrangement. The mechanism is integrated into the anthropomorphic upper body of the DLR David robot and responsible for the spinal rotation. Consequently, the actuator is crucial for the overall workspace of the robot and the realization of energy-efficient natural motions such as in dynamic running. The proposed hardware setup is experimentally validated in terms of the linearity in the spring characteristics, intrinsic damping, the excitation of resonance frequencies, and the ability to alter these resonance frequencies through stiffness adaptation during dynamic motions.
\end{abstract}

\section{INTRODUCTION}

In order to be properly operated in a human environment, modern robots must fulfill various requirements. Among others they have to be sufficiently robust with respect to impacts and collisions, and safety has to be ensured at all times. Designing the systems in a way such that they have similar kinematic and dynamic properties as humans (size, weight, two-handedness, kinematic redundancy, etc.) brings along many advantages and equips the robots with the ability to use common tools and manipulate objects and the environment in an efficient way. A high level of robustness is often achieved through the introduction of mechanical springs integrated in the actuated joints. Similar to muscle stiffening and loosening in human beings, variable stiffness actuators (VSA) can be integrated in robots [1]. Within the wide field of VSA, one has to distinguish between two main categories: on the one hand, non-linear spring elements are usually easier to design and realize in hardware, and the adaptability can be simply achieved by modification of the pretensioning, for example [2]-[4]. However, due to their non-linear nature it is rather difficult to model and master the complexity in the dynamic behavior. As a consequence, the rich field of linear oscillation theory cannot be applied. On the other hand, linear VSAs are usually more difficult to design but they have the advantage of simple modeling and the applicability of linear theory [5]. Especially in terms

\footnotetext{
${ }^{1}$ Authors are with the Institute of Robotics and Mechatronics, German Aerospace Center (DLR), Email: jens.reinecke@dlr.de

${ }^{2}$ Author is with the Robotic Systems Lab, ETH Zurich, Switzerland

This article is published by IEEE with Digital Object Identifier (DOI): 10.1109/LRA.2021.3117245
}

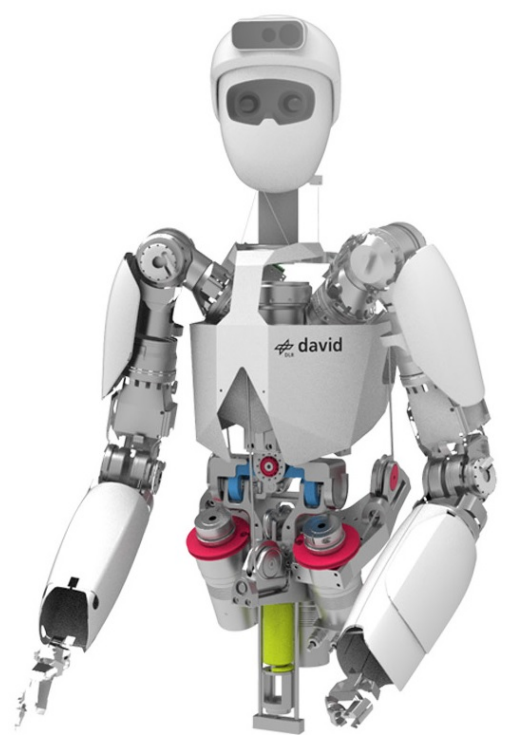

Fig. 1. CAD model of the upper body of the DLR David robot with the proposed torso joint

of the active excitation of resonance frequencies a linear behavior can be highly beneficial to improve the energy efficiency. Recent studies have confirmed that it can be difficult to bring nonlinear spring mechanisms into resonance properly [6].

\section{A. Natural and energy-efficient motions}

Nature demonstrates how animals can actively tune the natural frequency related to their locomotion system to reach high efficiency at various velocities [7]. The transfer to robotic systems has been undertaken multiple times already. In [8], the authors have shown that hopping in a range of speeds can be made efficient by means of variable stiffness components. An operation point for a robotic puppy has been searched in [9] to take advantage of the intrinsic resonance frequency. Similarly, the properties of the elastic potential have been exploited to improve the efficiency in hopping [10] and walking [11] in a humanoid robot, or jumping in a quadruped [12]. The authors in [13] have proposed an energy-efficient approach for locomotion of a compliant monopod system operated close to its natural frequency. While the above-mentioned works mainly focus on the legged subsystem, the underlying principles can also be applied to other components of the robot such as in the backbone [14] or in the arms [15]. 
B. Classification of robotic actuators with adaptable intrinsic stiffness

Since VSAs provide the means to actively adapt the intrinsic elasticities and natural frequencies, such mechatronic elements are often found in modern robots where energy efficiency, periodic motions, or robustness against impacts are the focus [16]. As explained in [1], the variability in the stiffness can be achieved in several ways. The most common ones are pretensioning of an effectively nonlinear spring, the modification of the physical properties of the spring, or variable transmission ratios. Although it is even possible to achieve linear characteristics with pretensioning, that is hard to realize in practice because quadratic springs are required [17], [18]. Mostly, an approximately linear behavior can be achieved in parts of the workspace, see Fig. 2 (a). The second category contains approaches to vary the effective spring length [18], [19] but the unused parts contribute to the overall weight, which is unfavorable in lightweight robotics.

In VSAs with variable transmission ratios, actuation designs have been proposed to shape the force-torquecharacteristic of serial elastic actuator (SEA) elements [20]. Extensions to concepts with the ability to adapt to the current load are presented in [21], [22]. The authors in [23] apply an actuator with variable transmission angle. While the observer behavior is dominated by frictional effects close to zero deflection angles, it becomes linear in the remaining workspace. An improved version is shown in [8], wherein friction effects are reduced and restricted to one single direction of deflection only. Several further variable-transmission concepts achieve a linear behavior in a region of the workspace through variable lever arms [24]-[26]. In [27], the energy-efficient and compact variable-lever arm mechanism AwAW-II has been proposed. By adjusting the position of the pivot point, the compliance can be specified as desired. Although the kinematics involve a non-linear behavior when considering the whole workspace, the assumption of linearity holds for small angles. The authors in [27] use torsional springs to realize the compliant behavior in a SEA structure, which is highly beneficial in terms of robustness against impacts. The DEA-based solution we propose in this work will rely on translational springs with subsequent conversion to a revolute joint by a rack-pinion connection. Also variable linear springs have been realized by means of variable transmission ratios which bring along all advantages of linear oscillation theory. While a fully linear spring mechanism has been proposed in [28], a similar design has been recently applied to damp a milling machine in an SEA configuration in [29]. In contrast to the latter use case where the spring mechanism has been exploited to damp out vibrations during milling, the mechanism presented in this work at hand is intended to deliberately excite resonance modes in a robot.

An alternative to SEAs are Differential Elastic Actuators (DEA). With similar performance as SEAs, one can integrate DEAs in a more compact way. both variable stiffness [30] and single stiffness [31]. In [32], different transmission ratios between load/motor and load/spring are implemented. As

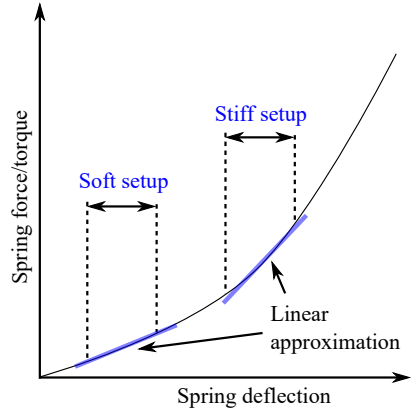

(a) Use of two areas in a non-linear spring

Fig. 2. Qualitative comparison between non-linear and linear VSAs, and the realization of the variable stiffness

a consequence, one can use springs with reduced stiffness compared to a classical SEA, which, in turn, is beneficial in terms of the quality in the joint torque measurement. Similar considerations have been conducted at the example of a pipeline inspection robot [33], to combine two identical motor units into one VSA [34], or to ease the cabeling for internal position sensors [35].

\section{Contributions}

In this article, we present a robotic joint for the spinal rotation of a humanoid upper body with adjustable linear spring characteristics (cf. Fig. 2(b)). To the best of our knowledge, this is the first of its kind. Compared to alternative variable-lever arm mechanisms that also adjust the position of the pivot point to change the joint stiffness [27], [29], our approach based on the original concept [28] has two main features.

1) We provide a completely linear spring characteristics in the whole workspace of the joint. That is highly beneficial in terms of the excitation of resonance modes, the improved capabilities to store energy in the spring, and the reduced modeling and control complexity.

2) The joint is arranged in a DEA setup instead of an SEA configuration. Since the spring mechanism is not part of the moving elements on the link side, the effective inertia to be accelerated is significantly reduced in order to strictly follow lightweight design principles.

This articulation for the spinal rotation complements the previously presented lower torso joints [36] of the DLR David robot. In practice, the specific setup is predestined for motions encountered during human-like walking and running [37]. Several experiments on the upper body of the robot validate the proposed mechanism and confirm the linear characteristics, the targeted setting and excitation of resonance frequencies, and the adjustment of the stiffness during highly dynamic motions.

\section{Structure}

The article is structured as follows: in Section II, the linear, adjustable spring mechanism is introduced and mathematically derived. Afterwards, the mechatronic design is described in Section III. The experimental validation is 


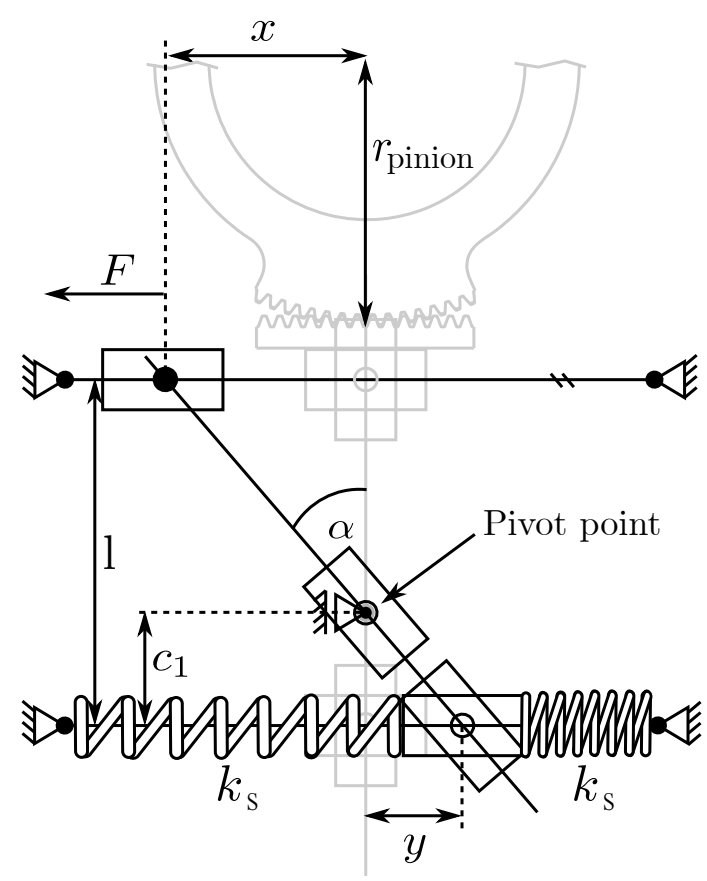

Fig. 3. Proposed mechanism with adaptable intrinsic stiffness, realized by shifting of the pivot point

conducted in Section IV, before the work is concluded in Section V.

\section{Adjustable Linear Spring Mechanism}

Figure 3 shows the schematic structure of the proposed mechanism. By moving the pivot point the transmission is actively modified. That is achieved through the adjustment of $c_{1} \in \mathbb{R}_{>0}$, where the range between $43.5 \mathrm{~mm}$ and $105 \mathrm{~mm}$ describes the practically relevant region. The constant distance of the two parallel linear guides is $l$ and the lever angle is $\alpha \in \mathbb{R}$. By means of the kinematic relations

$$
\begin{aligned}
& x=\left(l-c_{1}\right) \tan (\alpha) \\
& y=c_{1} \tan (\alpha)
\end{aligned}
$$

one can formulate the elastic potential $V \in \mathbb{R}_{\geq 0}$ wherein the overall stiffness $k$ includes both springs with a constant spring stiffness $k_{\mathrm{s}}$ illustrated in Fig. 3 such that $k=2 k_{\mathrm{s}}$ :

$$
V=\frac{1}{2} k y^{2}=k_{\mathrm{s}}\left(\frac{c_{1}}{l-c_{1}}\right)^{2} x^{2},
$$

which yields the spring force $F \in \mathbb{R}$

$$
F(x)=-\frac{\partial V}{\partial x}=-\underbrace{2 k_{\mathrm{s}}\left(\frac{c_{1}}{l-c_{1}}\right)^{2}}_{k_{\text {eff,trans }}} x .
$$

with the effective translational stiffness $k_{\text {eff,trans }} \in \mathbb{R}_{>0}$ of the mechanism. Since the considered joint in the robotic torso is of revolute type, the spring characteristics has to be transformed via the constant pinion radius $r_{\text {pinion }}$ as demonstrated in Fig. 3 (top).
The effective rotational stiffness $k_{\text {eff,rot }} \in \mathbb{R}_{>0}$ is consequently given by

$$
\begin{aligned}
k_{\text {eff }, \text { rot }} & =k_{\text {eff }, \text { trans }}\left(r_{\text {pinion }}\right)^{2} \\
& =2 k_{\mathrm{s}}\left(\frac{c_{1}}{l-c_{1}}\right)^{2}\left(r_{\text {pinion }}\right)^{2} .
\end{aligned}
$$

From (5) one can see the linear nature of the spring mechanism because $k_{\text {eff,rot }}$ stays constant for any fixed pivot point, that is, constant values of $c_{1}$. By shifting the pivot point, the stiffness can be arbitrarily varied. To obtain a desired effective rotational stiffness, one has to move the pivot point to

$$
c_{1}=\frac{l}{1+\sqrt{\frac{2 k_{\mathrm{s}}\left(r_{\text {pinion }}\right)^{2}}{k_{\text {eff }, \text { rot }}}}} .
$$

The main difference to a Continuous Variable Transmission based VSA is the fact that this mechanism has a limited range of motion due to its planar nature. In other words, the maximum amplitude is limited by the linear guide lengths. Depending on the pivot position this maximum is either reached by the link-side maximum rotation or the maximum spring deflection. That also implies a minimum effective stiffness to provide a proper range of motion in the joint. On the other side the stiffer the setting is, the more friction is present in the linear guides, thus the hysteresis will be increased.

Note that the actual transmission ratio of $101 / 100$ between the flex spline and the circular spline can be neglected in the model due to the approximation $101 / 100 \approx 1$.

\section{DESIGN}

The mechatronic design of the rotational joint is based on the requirement analysis of the torso of the David system [36]. In general, the considered torso must be able to withstand impacts and perform dynamic motions similar to the human counterpart. Considering the particular joint responsible for the spinal rotation, an oscillation at about $1.5 \mathrm{~Hz}$ is taken as nominal motion [37] with an overall target range of $0.5-3 \mathrm{~Hz}$. The total mass to be handled is about $30 \mathrm{~kg}$, consisting of the two arms with $13.7 \mathrm{~kg}$ each, the head of about $1.5 \mathrm{~kg}$, and the remaining structural elements which add up to about $0.9 \mathrm{~kg}$. Primarily the configuration of the arms defines the final inertia about this axis of motion. Correspondingly, the stiffness of the spring has been chosen in a way such that the resonance frequency of $1.5 \mathrm{~Hz}$ is achieved in the middle of the stiffness range in case of the nominal arm configuration illustrated in Fig. 10.

\section{A. Actuator design}

A 70x18 HWB Robodrive stock motor has been mounted in a star-parallel configuration, combined with RLS-Aksim RD-70 position sensors. The gearbox is a standard Harmonic Drive element of type HD-CPL-25-100 with a gear ratio of 100:1. That leads to a maximum nominal link-side speed of 42 turns $/ \mathrm{min}$. Such velocities are needed for the induced swinging. That also provides a sufficient margin for higher running velocities or the control of the spring at higher swinging frequencies. 
TABLE I

COMPARISON OF ROBUSTNESS AND DYNAMIC PROPERTIES

\begin{tabular}{|c||c|c|}
\hline & $\begin{array}{c}\text { Scenario: Impact } \\
\text { (inertia which is accelerated } \\
\text { through the gearbox) }\end{array}$ & $\begin{array}{c}\text { Scenario: Dynamic motions } \\
\text { (additional link-side inertia) }\end{array}$ \\
\hline Stiff setup & $9874 \mathrm{kgcm}^{2}$ & No additional inertia \\
\hline DEA setup & $20 \mathrm{kgcm}^{2}$ & $20 \mathrm{kgcm}^{2}$ \\
\hline SEA setup & No direct effect on gearbox & $92 \mathrm{kgcm}^{2}$ \\
\hline
\end{tabular}

\section{B. Differential design}

The adjustable spring mechanism is connected to the circular spline of the Harmonic Drive gearbox, see Fig. 4. The circular spline is guided by a thin ball bearing from Kaydon, connected to a gear wheel. The pinion wheel is connected to a gear rack which moves the linear guides of the adjustable spring mechanism, see Fig. 5. While this DEA arrangement tendentially leads to a lower robustness than classical SEA systems with spring between gearbox and link, the spring does not move along with the link. Consequently, that is beneficial in terms of lightweight and compact design as well as the dynamic capabilities of the torso. In a classical rigid design, the motor inertia is multiplied by the square of the gear ratio, which is avoided here. In Table I, two scenarios are compared for these three setups: the impact case and nominal dynamic motions. For the stiff setup, the proposed spring mechanism is consequently not used at all. The DEA setup is given by the design proposed in this article. If the proposed mechanism was serially mounted after the gearbox, the SEA setup would result. ${ }^{1}$ Table I demonstrates that the stiff setup is the lightest one, but any impact directly affects the gearbox and has to be counterbalanced by its internal elements, leading to an inferior robustness. In the SEA setup, the gearbox is not kinematically connected to the link-side but the impact will be completely counterbalanced by the spring mechanism. However, since the mechanism has to be mounted after the gearbox, a significantly increased link-side inertia results with $92 \mathrm{kgcm}^{2}$. The DEA setup describes a good compromise in terms of both aspects. The increased link-side inertia only amounts to about $20 \mathrm{kgcm}^{2}$, which is also a small inertia to be accelerated through the gearbox during an impact. Additionally, the DEA provides more design freedom.

The intended ways of mounting of the Harmonic Drive gearbox [38] do not contain a DEA setup. Although the bearing clearances specified by the manufacturer have been taken into account during the design proposed here, the question of the life cycle should be raised. Nevertheless, previous experiences with DEA setups have confirmed a good long-term use. The BAVs joint [39], for example, is arranged in a DEA structure and used in the arms of

${ }^{1}$ The values in Table I relate to the linearized system with the pivot point at $74.25 \mathrm{~mm}$. The inertia is computed from the CAD model. The high inertia difference in the impact case originates when the inertia is multiplied by the square of the gear-ratio, which is $100^{2}$ in the stiff setup compared to $1.01^{2}$ in the DEA setup.

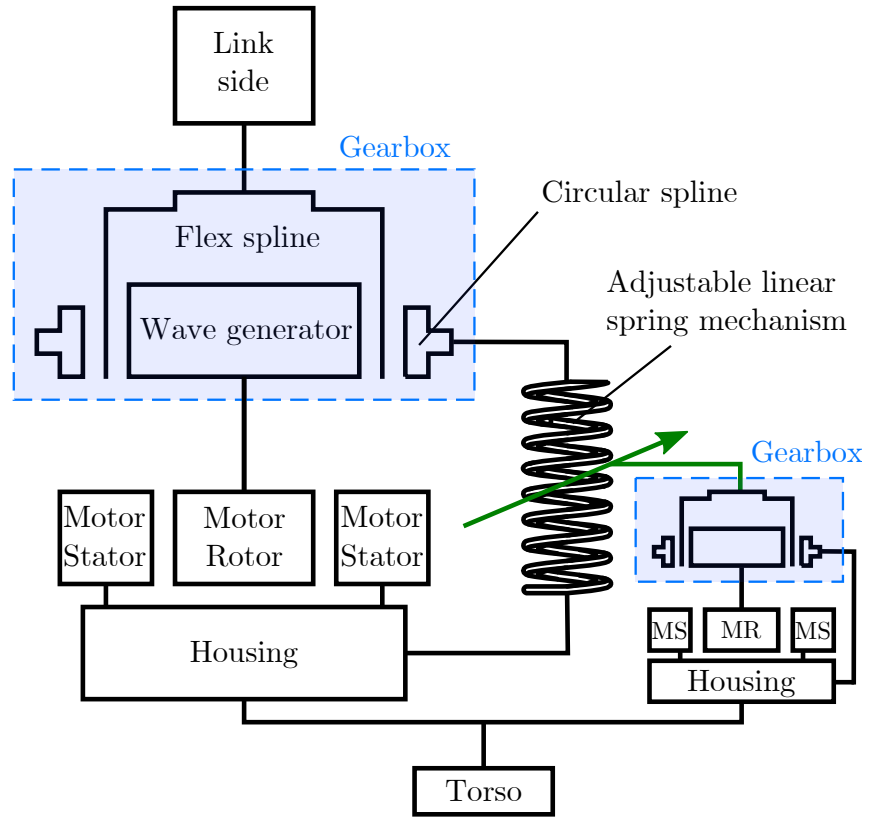

Fig. 4. DEA structure of the proposed torso joint: The spring mechanism is connected via a rack-pinion connection to the circular spline of the gearbox. The pivot point of the spring mechanism is connected to the housing.

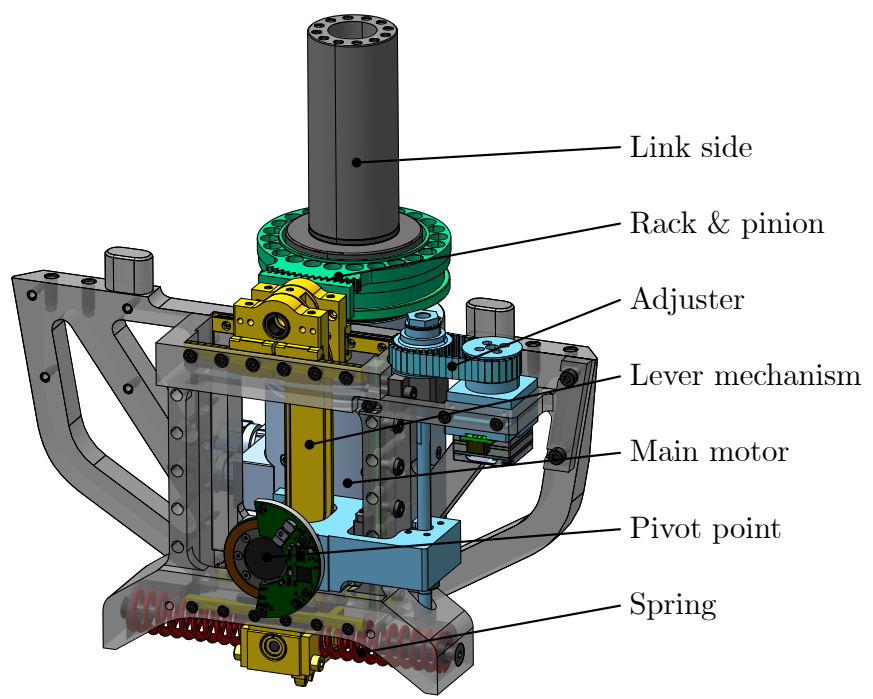

Fig. 5. Mechatronic design of the proposed torso joint

the David robot for several years without any problems in practice.

\section{Spring mechanism design}

The linear guides are off-the-shelf components built by Nippon Bearing and feature small sliding blocks predestined for this purpose. In the pivot point, needle bearings are integrated to absorb the radial forces. In contrast to comparable ball bearings, they require less space and can support higher loads. The bearings are selected to withstand also twice the stiffness of the nominal/used springs. No noteworthy axial forces occur at the pivot point. Thus, an axial needle roller bearing as thrust washer has been chosen. Although that 
leads to more friction than in axial ball bearing washers, the reduction of the integration space is significant. The linearization in the spring characteristics necessitates several linear guides. Although these feature low intrinsic friction, there is a load-dependent friction component. Especially in stiff settings, the influence of hysteresis will be more apparent. That will be investigated in Sec. IV-A. Moreover, the effect of friction in the entire mechanism will be evaluated in a second experiment in Sec. IV-B, where the decay rate and damping ratio are identified for different adjuster positions.

The springs with $18.1 \mathrm{~N} / \mathrm{mm}$ stiffness have the same size allowing us to select two different range corridors for the torso. Thus we can adjust to the setup with one or two arms, respectively.

The experiments in Sec. IV are conducted with springs of $9.8 \mathrm{~N} / \mathrm{mm}$ stiffness as the robot is currently equipped with only one arm so far. The stiffness is adjusted by an ILM25x8 Robodrive motor with $\omega_{m}=24000$ turns $/ \mathrm{min}$ and a Harmonic Drive gearbox with gear ratio of $i_{\text {hd }}=100$. The ball screw of the spindle is connected via a belt with a ratio of $i_{\text {belt }}=25 / 32$. Since the spindle slope is $p_{\text {spindle }}=1 \frac{\mathrm{mm}}{\text { turn }}$ the maximum adjustment speed of the system is

$$
v_{\text {max }}=i_{\text {belt }} \frac{\omega_{m}}{i_{\text {hd }}} p_{\text {spindle }}=3.125 \mathrm{~mm} / \mathrm{s} \text {. }
$$

\section{VALIDATION}

This section addresses the experimental validation of the proposed mechanism in the torso of the David robot.

\section{A. Linearity and adjustment validation}

The stiffness adjustment and the linearity in the characteristics are confirmed in static experiments. The link side is rigidly connected to an external force sensor, while the motor is slowly moved such that the springs get deflected. From now on, the motor position describes the motor variable that has already been kinematically converted to link-side dimensions through multiplication by the gear ratio 1:100. In total, 10 different pivot points are evaluated, where each setting is sampled within a time window of about 30 s covering 90000 measurements. In Fig. 6 and Table II the feasible stiffness ranges are shown for the $9.8 \mathrm{~N} / \mathrm{mm}$ spring. One can identify the existence of hysteresis effects which primarily appear in higher-stiffness settings, since higher loads in the bearings and the linear guides result in larger frictional effects. Given the inertia of the upper body, the resonance frequencies of a comparable linear mass-spring-damper system lie in the range of $0.5-3 \mathrm{~Hz}$. That corresponds to running velocities up to $10 \mathrm{~km} / \mathrm{h}$ representing common values in humans [37]. The bottom diagram in Fig. 6 depicts the relative error in the achieved stiffness, that is, based on the deviation between modeled and measured stiffness. Note that one could optionally precompensate for such reproducible errors.

\section{B. Damping validation}

The intrinsic damping is investigated in the following experiments. All VSA joints in the arm are set as stiff as possible, and the joint configuration is chosen such that internal
TABLE II

MODELED AND MEASURED STIFFNESS IN THE EXPERIMENT OF FIG. 6

\begin{tabular}{|c|c|c|c|}
\hline Setting & $c_{1}$ & Stiffness (measured) & Stiffness (modeled) \\
\hline \hline 1 & $43.5 \mathrm{~mm}$ & $8.9 \mathrm{Nm} / \mathrm{rad}$ & $9.0 \mathrm{Nm} / \mathrm{rad}$ \\
2 & $50.5 \mathrm{~mm}$ & $13.5 \mathrm{Nm} / \mathrm{rad}$ & $13.9 \mathrm{Nm} / \mathrm{rad}$ \\
3 & $57.0 \mathrm{~mm}$ & $19.7 \mathrm{Nm} / \mathrm{rad}$ & $20.3 \mathrm{Nm} / \mathrm{rad}$ \\
4 & $64.0 \mathrm{~mm}$ & $29.0 \mathrm{Nm} / \mathrm{rad}$ & $29.9 \mathrm{Nm} / \mathrm{rad}$ \\
5 & $71.0 \mathrm{~mm}$ & $41.7 \mathrm{Nm} / \mathrm{rad}$ & $43.6 \mathrm{Nm} / \mathrm{rad}$ \\
6 & $77.6 \mathrm{~mm}$ & $59.7 \mathrm{Nm} / \mathrm{rad}$ & $62.0 \mathrm{Nm} / \mathrm{rad}$ \\
7 & $84.5 \mathrm{~mm}$ & $85.1 \mathrm{Nm} / \mathrm{rad}$ & $89.9 \mathrm{Nm} / \mathrm{rad}$ \\
8 & $91.4 \mathrm{~mm}$ & $123.2 \mathrm{Nm} / \mathrm{rad}$ & $131.3 \mathrm{Nm} / \mathrm{rad}$ \\
9 & $98.0 \mathrm{~mm}$ & $174.3 \mathrm{Nm} / \mathrm{rad}$ & $191.5, \mathrm{Nm} / \mathrm{rad}$ \\
10 & $104.9 \mathrm{~mm}$ & $256.8 \mathrm{Nm} / \mathrm{rad}$ & $292.1 \mathrm{Nm} / \mathrm{rad}$ \\
\hline
\end{tabular}
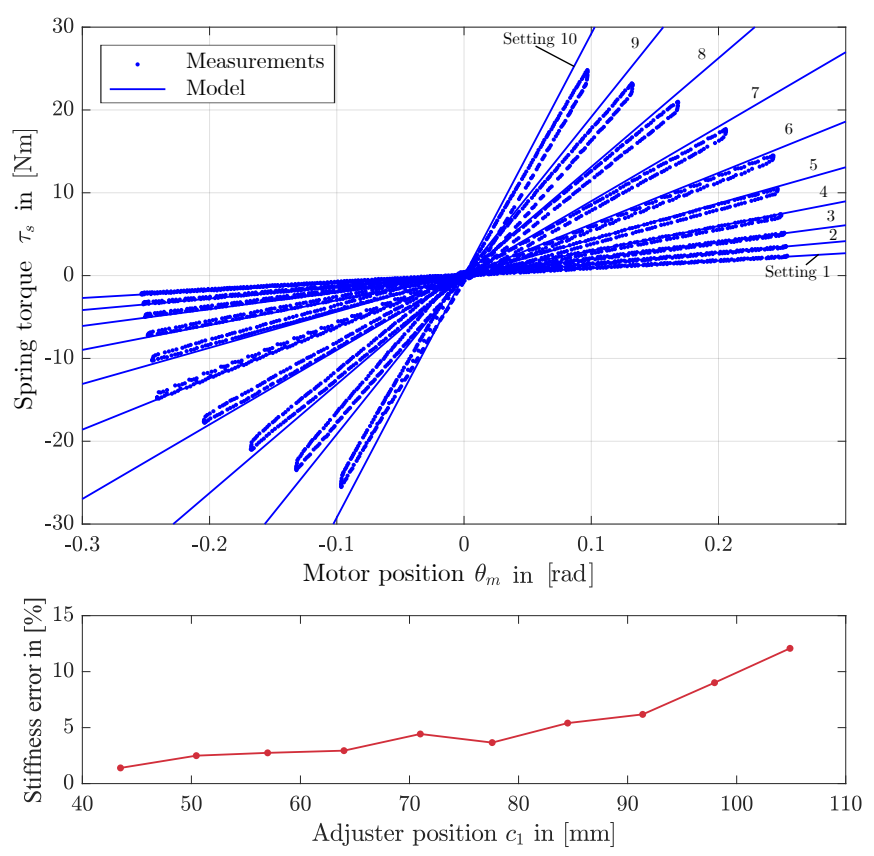

Fig. 6. Measurement of ten settings from Table II with different adjuster positions. Each of these settings has been evaluated in 90000 measurements recorded in about $30 \mathrm{~s}$. The effect of hysteresis increases for increasing stiffness, due to the larger loads present in the bearings. All settings demonstrate that the desired linear behavior is adequately achieved.

arm motions are largely avoided. Moreover, the motor side is controlled by a stiff PD controller to keep the zero position, and the link is manually deviated. Then the link is released and the transient is observed. The results are shown in Fig. 7. The top diagram depicts one exemplary scenario, where the pivot point is set to $78.7 \mathrm{~mm}$. An exponential function is fitted to conclude the decay rate (bottom diagram). The decay rate indicates that friction in low and high stiffness settings has a higher influence. In addition, the second diagram in Fig. 7 shows the damping ratio.

\section{Resonance frequencies}

In the following dynamic experiment, the robotic arms of David are replaced by weight handles, resulting in constant inertia with respect to the considered torso joint axis. The weights are chosen such that the overall inertia reflects values 

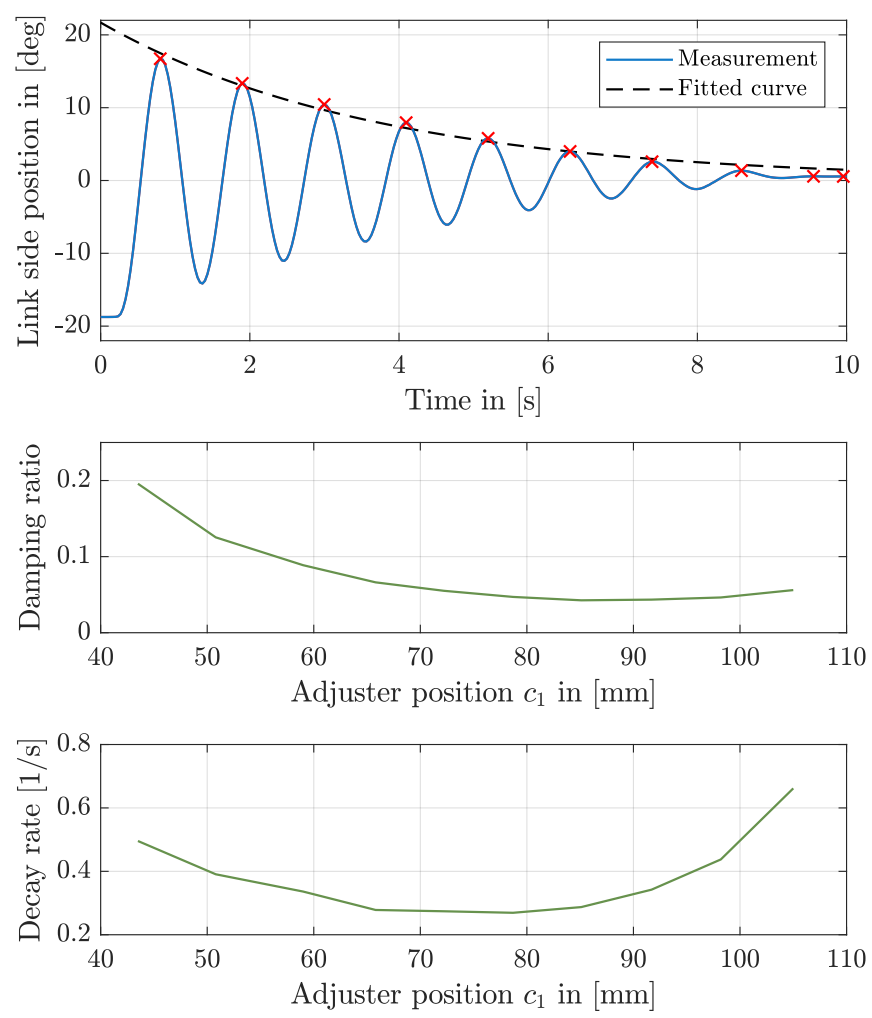

Fig. 7. Damping experiment, Top: Exemplary measurement at adjuster position $78.7 \mathrm{~mm}$, Middle: Damping ratio depending on the adjuster position, Bottom: Decay Rate depending on the adjuster position

in human-like running. The motivation is to investigate relevant resonance frequencies on the hardware.

A sweep signal is applied to stiff motor-PD control as desired motor-side position. The reference amplitude varies between $1.25-2^{\circ} .^{2}$ The sweep covers frequencies between $0.15-4.5 \mathrm{~Hz}$, therefore including the practically relevant range discussed in Sect. IV-A.

Figure 8 depicts the measured amplifications, namely the link-side positions divided by the motor-side positions, depending on the adjuster position and the excitation frequency. One can observe the shifting of the resonance frequencies through the placement of the pivot point. These experimentally identified resonance frequencies are isolated and plotted in Fig. 9 (left).

\section{Online-adaption of the stiffness}

In the following dynamic experiment the stiffness adjustment is evaluated during motion. One articulated arm is mounted and the joint configuration is chosen such that it reflects the natural one during human running. The scenario starts when the mechanism is in resonance with maximum amplitude. Then the pitch-axis in the shoulder joint is rotated by $20^{\circ}$ such that the inertia about the considered torso axis increases, see Fig. 10. This action consequently results in a smaller amplitude. In order to get into resonance again, the

\footnotetext{
${ }^{2}$ The amplitude is variable to allow large link-side motions under the constraint of limited admissible spring deflections depending on the adjuster position.
}

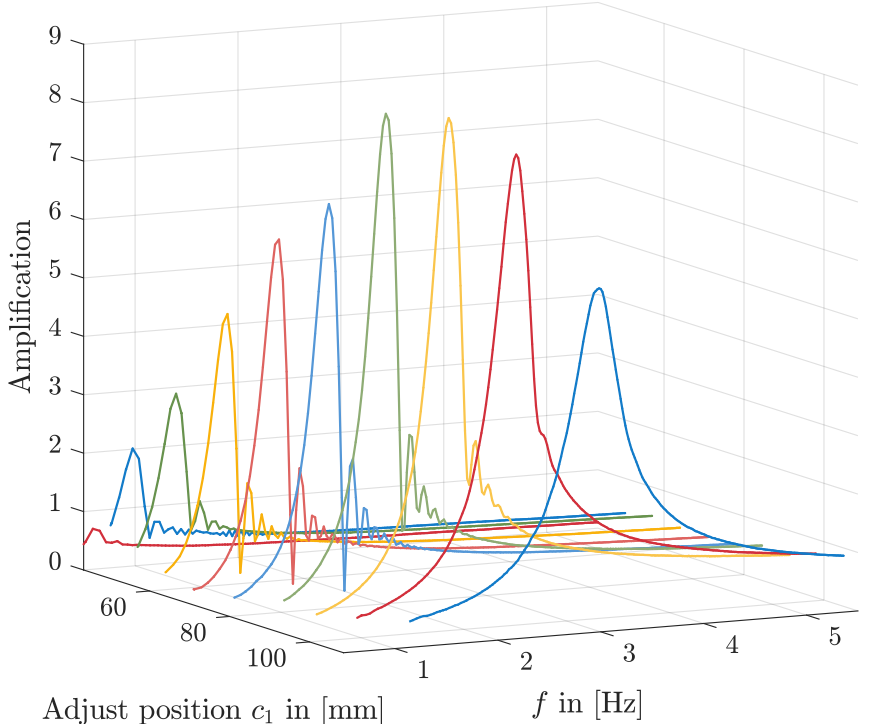

Fig. 8. Measured amplifications (link-side position divided by motor-side position) for different adjuster positions and frequencies with fixed and constant inertia. The motor-side position is the motor angle transformed to link-side-equivalent dimensions by means of the gear ratio.

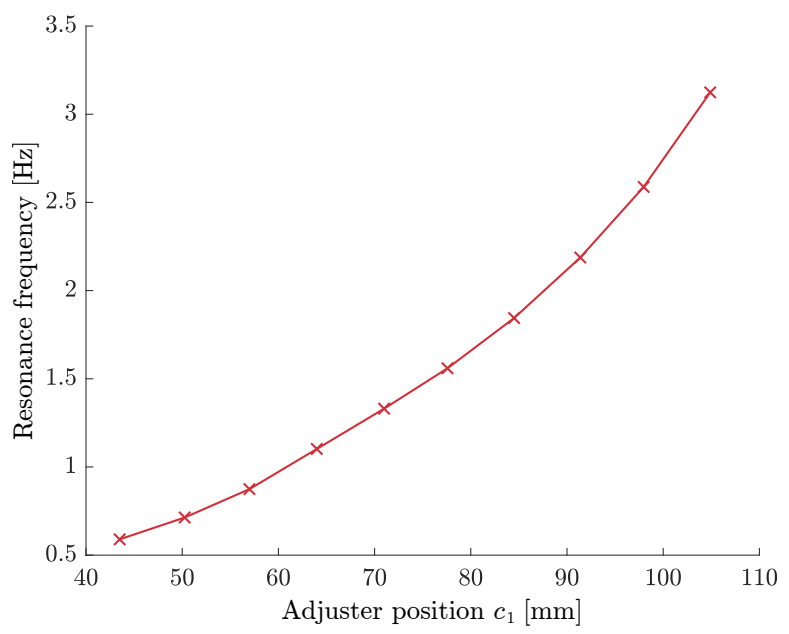

Fig. 9. Resonance frequencies for different adjuster positions for a fixed inertia mounted on the link side

adjuster is actuated and the pivot point is moved. The results are shown in Fig. 11 and in the accompanying video.

\section{CONCLUSIONS}

In this work we presented a variable-stiffness actuator with linear characteristics integrated in a robotic torso in a differential arrangement. The model and key parameters were discussed to adapt the system for different applications and scenarios. We presented the actuator and mechanism design and the realization in the humanoid robot David. The hardware was validated in terms of linearity, dynamic behavior, and resonance adaption for typical running motions. The experiments confirmed the assumption of a linear spring behavior with adaptable stiffness, and they demonstrated that resonance frequencies can be both deliberately excited and actively adapted during highly dynamic motions. 


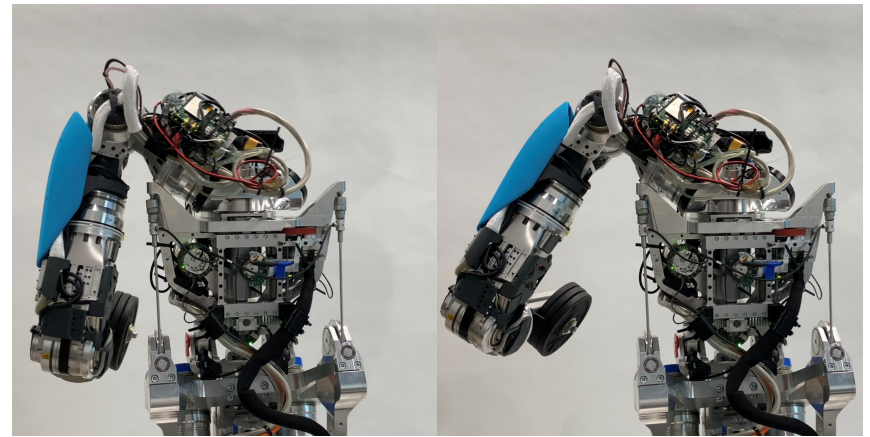

Fig. 10. Arm configurations of the upper body. Left: starting configuration. Right: end configuration. The pitch-axis in the shoulder is actuated to increase the joint position by $20^{\circ}$.
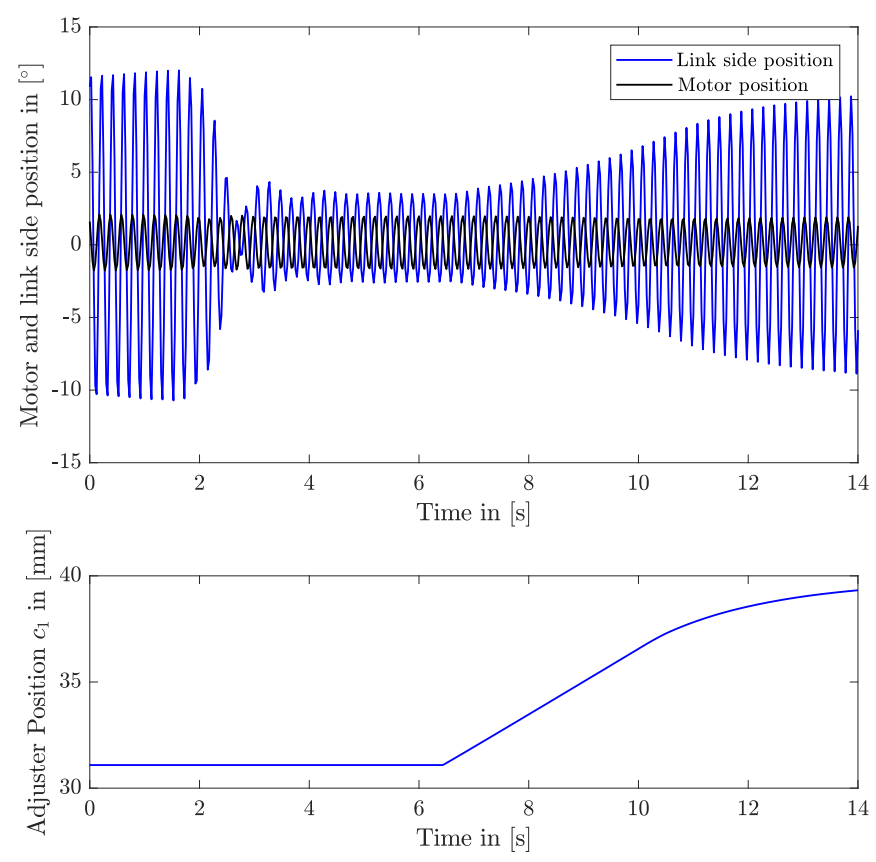

Fig. 11. The upper body starts with an oscillation at a frequency of about $1.5 \mathrm{~Hz}$ with an amplitude of about $11^{\circ}$. After increasing the inertia at $t \approx 2 \mathrm{~s}$, as illustrated in Fig. 10, the spring oscillation is reduced. By adjusting the stiffness, the resonance oscillation can be obtained again.

\section{ACKNOWLEDGMENT}

The authors would like to thank Annika Junger who helped to build the model with linear guides, and Haddar Sleiman who designed a first prototype for functional tests. This work was supported in part by the German Research Foundation (DFG) under Grant 405032572 and the ERC Advanced Grant M-RUNNERS (Project ID 835284).

\section{REFERENCES}

[1] B. Vanderborght et al., "Variable impedance actuators: A review," Robotics and Autonomous Systems, vol. 61, no. 12, pp. 1601-1614, Dec. 2013.

[2] J. Park, H. Kim, and J. Song, "Safe robot arm with safe joint mechanism using nonlinear spring system for collision safety," in IEEE International Conference on Robotics and Automation (ICRA), May 2009, pp. 3371-3376.
[3] W. Friedl, H. Höppner, F. Petit, and G. Hirzinger, "Wrist and forearm rotation of the DLR hand arm system: Mechanical design, shape analysis and experimental validation," in IEEE/RSJ International Conference on Intelligent Robots and Systems (IROS), Sep. 2011, pp. $1836-1842$.

[4] B. Vanderborght, N. G. Tsagarakis, R. Van Ham, I. Thorson, and D. G. Caldwell, "MACCEPA 2.0: compliant actuator used for energy efficient hopping robot Chobino1D," Autonomous Robots, vol. 31, no. 1, p. 55, Apr. 2011.

[5] S. Savin, R. Khusainov, and A. Klimchik, "Control of actuators with linearized variable stiffness," in 9th IFAC Conference on Manufacturing Modelling, Management and Control (MIM), vol. 52, no. 13, 2019, pp. 713-718.

[6] D. Lakatos, F. Petit, and A. Albu-Schaffer, "Nonlinear oscillations for cyclic movements in human and robotic arms," IEEE Transactions on Robotics, vol. 30, no. 4, pp. 865-879, Aug. 2014.

[7] B. K. Ahlborn, R. W. Blake, and W. M. Megill, "Frequency tuning in animal locomotion," Zoology, vol. 109, no. 1, pp. 43-53, Feb. 2006.

[8] H. Q. Vu, X. Yu, F. Iida, and R. Pfeifer, "Improving energy efficiency of hopping locomotion by using a variable stiffness actuator," IEEE/ASME Transactions on Mechatronics (TMECH), vol. 21, no. 1, pp. 472-486, Feb. 2016.

[9] J. Buchli, F. Iida, and A. Ijspeert, "Finding resonance: Adaptive frequency oscillators for dynamic legged locomotion," in IEEE/RSJ International Conference on Intelligent Robots and Systems (IROS). IEEE, Oct. 2006, pp. 3903-3909.

[10] B. Ugurlu, J. A. Saglia, N. G. Tsagarakis, and D. G. Caldwell, "Hopping at the resonance frequency: A trajectory generation technique for bipedal robots with elastic joints," in IEEE International Conference on Robotics and Automation (ICRA). IEEE, May 2012, pp. 14361443.

[11] F. L. Moro, N. G. Tsagarakis, and D. G. Caldwell, "Efficient humanlike walking for the COmpliant huMANoid COMAN based on kinematic motion primitives (kMPs)," in IEEE International Conference on Robotics and Automation (ICRA), May 2012, pp. 2007-2014.

[12] D. Lakatos, G. Garofalo, A. Dietrich, and A. Albu-Schaffer, "Jumping control for compliantly actuated multilegged robots," in IEEE International Conference on Robotics and Automation (ICRA), 2014, pp. $4562-4568$.

[13] F. Guenther and F. Iida, "Energy-efficient monopod running with a large payload based on open-loop parallel elastic actuation," IEEE Transactions on Robotics, vol. 33, no. 1, pp. 102-113, Feb. 2017.

[14] E. Parra Ricaurte, J. Colorado, S. Dominguez, and C. Rossi, "A bioinspired quasi-resonant compliant backbone for low power consumption quadrupedal locomotion:," in 17th International Conference on Informatics in Control, Automation and Robotics (ICINCO), 2020, pp. 242-249.

[15] D. Lakatos, M. Görner, F. Petit, A. Dietrich, and A. Albu-Schäffer, "A modally adaptive control for multi-contact cyclic motions in compliantly actuated robotic systems," in IEEE/RSJ International Conference on Intelligent Robots and Systems (IROS), Nov 2013, pp. 5388-5395.

[16] F. Petit, A. Dietrich, and A. Albu-Schäffer, "Generalizing Torque Control Concepts: Using Well-Established Torque Control Methods on Variable Stiffness Robots," IEEE Robotics \& Automation Magazine, vol. 22, no. 4, pp. 37-51, December 2015.

[17] C. E. English and D. Russell, "Mechanics and stiffness limitations of a variable stiffness actuator for use in prosthetic limbs," Mechanism and Machine Theory, p. 19, 1999.

[18] A. Jafari, H. Q. Vu, and F. Iida, "Determinants for stiffness adjustment mechanisms," Journal of Intelligent \& Robotic Systems, vol. 82, no. 3, pp. 435-454, Jun. 2016.

[19] K. W. Hollander, T. G. Sugar, and D. E. Herring, "Adjustable robotic tendon using a 'jack spring," in IEEE 9th International Conference on Rehabilitation Robotics (ICORR), Jun. 2005, pp. 113-118.

[20] S. Stramigioli, G. v. Oort, and E. Dertien, "A concept for a new energy efficient actuator," in IEEE/ASME International Conference on Advanced Intelligent Mechatronics (AIM), Jul. 2008, pp. 671-675.

[21] L. Mooney and H. Herr, "Continuously-variable series-elastic actuator," in IEEE 13th International Conference on Rehabilitation Robotics (ICORR), Jun. 2013, pp. 1-6.

[22] S. Kim, J. Sim, and J. Park, "Elastomeric continuously variable transmission combined with twisted string actuator," IEEE Robotics and Automation Letters, vol. 5, no. 4, pp. 5477-5484, Oct. 2020.

[23] H. V. Quy, L. Aryananda, F. I. Sheikh, F. Casanova, and R. Pfeifer, "A novel mechanism for varying stiffness via changing transmission 
angle," in IEEE International Conference on Robotics and Automation (ICRA), May 2011, pp. 5076-5081.

[24] S. S. Groothuis, G. Rusticelli, A. Zucchelli, S. Stramigioli, and R. Carloni, "The variable stiffness actuator vsaUT-II: Mechanical design, modeling, and identification," IEEE/ASME Transactions on Mechatronics, vol. 19, no. 2, pp. 589-597, Apr. 2014.

[25] N. G. Tsagarakis, I. Sardellitti, and D. G. Caldwell, "A new variable stiffness actuator (CompAct-VSA): Design and modelling," in IEEE/RSJ International Conference on Intelligent Robots and Systems (IROS), Sep. 2011, pp. 378-383.

[26] B. Zhao, H. Zhu, Y. Zhong, and Y. Guan, "Design and modeling of a module with locally linear variable stiffness," in 2018 IEEE International Conference on Robotics and Biomimetics (ROBIO), Dec. 2018, pp. 2038-2044.

[27] A. Jafari, N. G. Tsagarakis, and D. G. Caldwell, "AwAS-II: A new actuator with adjustable stiffness based on the novel principle of adaptable pivot point and variable lever ratio," in IEEE International Conference on Robotics and Automation (ICRA). Shanghai, China: IEEE, May 2011, pp. 4638-4643.

[28] J. Reinecke and A. Dietrich, "Federmechanismus," DE patent DE102 015206811 A1, Oct., 2016.

[29] N.-D. Vuong, R. Li, C.-M. Chew, A. Jafari, and J. Polden, "A novel variable stiffness mechanism with linear spring characteristic for machining operations," Robotica, vol. 35, no. 7, pp. 1627-1637, Jul. 2017.

[30] S. Wolf and G. Hirzinger, "A new variable stiffness design: Matching requirements of the next robot generation," in IEEE International Conference on Robotics and Automation (ICRA). Pasadena, CA, USA: IEEE, May 2008, pp. 1741-1746.

[31] M. Lauria, M. Legault, M. Lavoie, and F. Michaud, "Differential elastic actuator for robotic interaction tasks," in IEEE International Conference on Robotics and Automation (ICRA), May 2008, pp. 36063611.

[32] C. Lee and S. Oh, "Configuration and performance analysis of a compact planetary geared elastic actuator," in IECON 2016 - 42nd Annual Conference of the IEEE Industrial Electronics Society, Oct. 2016, pp. 6391-6396.

[33] A. Kakogawa and S. Ma, "A differential elastic joint for multi-linked pipeline inspection robots," in IEEE/RSJ International Conference on Intelligent Robots and Systems (IROS), Oct. 2018, pp. 949-954.

[34] R. Wang and H. Huang, "ADEA — active variable stiffness differential elastic actuator : Design and application for safe robotics," in IEEE International Conference on Robotics and Biomimetics (ROBIO), Dec. 2011, pp. 2768-2773.

[35] P. Herodotou and S. Wang, "Design, modelling, and experimental evaluation of a compact elastic actuator for a gait assisting exoskeleton," in IEEE 16th International Conference on Rehabilitation Robotics (ICORR), Jun. 2019, pp. 331-336.

[36] J. Reinecke, B. Deutschmann, A. Dietrich, and M. Hutter, "An anthropomorphic robust robotic torso for ventral/dorsal and lateral motion with weight compensation," IEEE Robotics and Automation Letters, vol. 5, no. 3, pp. 3876-3883, Jul. 2020.

[37] A. Thorstensson, J. Nilsson, H. Carlson, and M. Zomlefer, "Trunk movements in human locomotion," Acta Physiologica Scandinavica, vol. 121, no. 1, pp. 9-22, 1984.

[38] "Engineering data catalogue," Harmonic Drive AG, Tech. Rep., 2017.

[39] H. Höppner, W. Wiedmeyer, and P. van der Smagt, "A new biarticular joint mechanism to extend stiffness ranges," in 2014 IEEE International Conference on Robotics and Automation (ICRA), 2014, pp 3403-3410. 